\title{
Comparison of crude extract from durio zibethinus M. (durian) leaf waste via ultrasound-assisted extraction and accelerated solvent extraction: antioxidant activity and cytotoxicity
}

\begin{abstract}
The objective of this study was to compare the antioxidant activity and cytotoxicity of Durio zibethinus M. (Durian) leaf extract from two extraction methods. Ultrasound-assisted extraction and Accelerated-solvent extraction were used to produce crude extract. The results revealed that UAE achieved $3 \times$ higher in total phenolic content in the leaf extract compared to ASE. DPPH radical scavenging activity was $4.6 \times$ higher in leaf extract from ASE. No significant differences reported in ferric reducing power, and total flavonoid content of the leaf extract between the two methods. Cytotoxicity via MTT assay demonstrated no significant differences in cell viability upon exposure to the leaf extract from both methods. This suggested that they were appropriate in producing Durio zibethinus M. leaf extract for end use application in food related product. Both ensured similar level of safety in Durio zibethinus M. leaf extract as a new potential ingredient for the food industry.
\end{abstract}

Keyword: Accelerated solvent extraction; Antioxidant activity; Cytotoxicity; Durio zibethinus M. leaf; Ultrasound-assisted extraction 